\title{
Solution of the Cauchy Problem for a Hyperbolic Equation with Constant Coefficients in the Case of Two Independent Variables
}

\author{
V. I. Korzyuk and I. S. Kozlovskaya \\ Institute of Mathematics, National Academy of Sciences, Minsk, Belarus
}

Received April 25, 2011

\begin{abstract}
On the plane, we consider a linear partial differential equation of arbitrary order of hyperbolic type. The operator in the equation is a composition of first-order differential operators. The equation is accompanied with Cauchy conditions. For the equation, we obtain an analytic form of the general solution, from which we single out the unique classical solution of the Cauchy problem.
\end{abstract}

DOI: $10.1134 /$ S0012266112050096

\section{INTRODUCTION}

In the present paper, we obtain an analytic form of the solution of the Cauchy problem for an $m$ th-order hyperbolic equation with constant coefficients, where $m$ is a positive integer. The operator in the equation is a composition of first-order differential operators. In the Cauchy problem, the unknown function depends on two independent variables.

By using the characteristics of the equation, we determine its general solution. From the general solution, we single out the solution that satisfies the Cauchy conditions.

The d'Alembert formula [1, p. 294; 2, pp. 54-56] is one of first results of the method of characteristics. Note that the method of characteristics permits one to find the general solution and the solution of the Cauchy problem for a linear first-order partial differential equation [3, pp. 539-554; 4, pp. 306-343].

This method was used for finding solutions of the Cauchy problem for many other differential equations, including nonlinear ones, in particular, for Hamilton-Jacobi equations, first-order quasilinear equations, etc. [5].

The Cauchy problem for hyperbolic partial differential equations was considered by other methods as well, mainly, by methods of functional analysis. But they do not permit one to construct solutions in analytic form, and only the well-posed statement of the problem can be proved [6-15]. Note also the paper [16] in which analytic forms of the general solution for partial differential equations and solutions of the Cauchy problem were constructed with the use of computer algebra systems.

\section{STATEMENT OF THE PROBLEM}

We study a problem on the plane $\mathbb{R}^{2}$ of two independent variables $t$ and $x$. For short, we use the following notation of partial derivatives: $\partial_{t}=\partial / \partial t, \partial_{x}=\partial / \partial x, \partial_{t}^{j}=\partial^{j} / \partial t^{j}, \partial_{x}^{j}=\partial^{j} / \partial x^{j}$, and $\partial_{t}^{s} \partial_{x}^{j}=\partial^{s+j} / \partial t^{s} \partial x^{j}$. In the domain $Q=(0, \infty) \times \mathbb{R}$, consider the $m$ th-order differential equation

$$
\mathfrak{L}^{(m)} u=\prod_{k=1}^{m}\left(\partial_{t}-a^{(k)} \partial_{x}+b^{(k)}\right) u(t, x)=f(t, x), \quad(t, x) \in Q,
$$


where $a^{(k)}$ and $b^{(k)}(k=1, \ldots, m)$ are coefficients in $\mathbb{R}$ (real numbers) and $f: \mathbb{R}^{2} \supset Q \ni(t, x) \rightarrow$ $u(t, x) \in \mathbb{R}$ is a function defined in $Q$. The following Cauchy conditions are imposed on the boundary $\partial Q=\{(t, x) \in \bar{Q} \mid t=0\}$ of the domain $Q$ :

$$
\left.\partial_{t}^{j} u\right|_{t=0}=d^{j} \varphi^{(j)}(x), \quad x \in \mathbb{R}, \quad j=0, \ldots, m-1 ;
$$

here $\partial_{t}^{0} u=u, \bar{Q}$ is the closure of the domain $Q, \bar{Q}=[0, \infty) \times \mathbb{R},[0, \infty)=(0, \infty) \cup\{0\}$, and $\{0\}$ is the singleton consisting of zero.

\section{GENERAL SOLUTION OF THE HOMOGENEOUS EQUATION}

Consider the homogeneous equation (2.1), that is, the equation

$$
\mathfrak{L}^{(m)} u=0, \quad(t, x) \in Q .
$$

We set

$$
w(t, x)=\prod_{k=1}^{m-1}\left(\partial_{t}-a^{(k)} \partial_{x}+b^{(k)}\right) u .
$$

For the function $w$, Eq. (3.1) is the first-order differential equation

$$
\partial_{t} w-a^{(m)} \partial_{x} w+b^{(m)} w=0 .
$$

By using the change of variables

$$
w(t, x)=e^{-b^{(m)} t} v(t, x),
$$

one can reduce Eq. (3.2) to the equation

$$
\partial_{t} v-a^{(m)} \partial_{x} v=0
$$

for the function $v$.

It is known that the equation of characteristics for (3.4) has the form

$$
d x+a^{(m)} d t=0,
$$

and its general solution can be represented by the formula $x+a^{(m)} t=C^{(m)}$, where $C^{(m)}$ is an arbitrary real constant.

By making the change of independent variables

$$
\xi=x+a^{(m)} t+C^{(m)}, \quad \eta=t,
$$

one can reduce Eq. (3.4) to the equation

$$
\partial_{\eta} \tilde{v}=0
$$

for the function $\tilde{v}(\xi, \eta)=v(t, x)$. Hence the general solution of Eq. (3.6) is given by the expression

$$
\tilde{v}(\xi, \eta)=g^{(m)}(\xi),
$$

where $g^{(m)}$ is an arbitrary differentiable function. By returning to the independent variables $t$ and $x$ with the use of the change of variables (3.5) and by using relation (3.3), we obtain the general solution of Eq. (3.2) in the form

$$
w(t, x)=e^{-b^{(m)} t} g^{(m)}\left(x+a^{(m)} t+C^{(m)}\right) .
$$

By using definition (3.3) of the function $w$, from Eq. (3.1), we obtain the $(m-1)$ st-order differential equation

$$
\mathfrak{L}^{(m-1)} u=\prod_{k=1}^{m-1}\left(\partial_{t}-a^{(k)} \partial_{x}+b^{(m)}\right) u=e^{-b^{(m)} t} g^{(m)}\left(x+a^{(m)} t+C^{(m)}\right) .
$$


Now consider Eq. (3.9). By introducing the notation

$$
w(t, x)=\prod_{k=1}^{m-2}\left(\partial_{t}-a^{(k)} \partial_{x}+b^{(k)}\right) u
$$

again, we rewrite Eq. (3.9) in the form

$$
\partial_{t} w-a^{(m-1)} \partial_{x} w+b^{(m-1)} w=e^{-b^{(m)}} t g^{(m)}\left(x+a^{(m)} t+C^{(m)}\right) .
$$

The change of variables

$$
w(t, x)=e^{-b^{(m-1)} t} v(t, x)
$$

reduces Eq. (3.11) to the equation

$$
\partial_{t} v-a^{(m-1)} \partial_{x} v=e^{\left(b^{(m-1)}-b^{(m)}\right) t} g^{(m)}\left(x+a^{(m)} t+C^{(m)}\right)
$$

for $v$.

By making the change of independent variables

$$
\xi=x+a^{(m-1)} t+C^{(m-1)}, \quad \eta=t
$$

in Eq. (3.12), where $C^{(m-1)}$ is an arbitrary constant, we reduce it to the form

$$
\partial_{\eta} \tilde{v}=e^{\left(b^{(m-1)}-b^{(m)}\right) \eta} g^{(m)}\left(\xi+\left(a^{(m)}-a^{(m-1)}\right) \eta+C\right) .
$$

The general solution of Eq. (3.14) is given by the formula

$$
\tilde{v}(\xi, \eta)=\int e^{\left(b^{(m-1)}-b^{(m)}\right) \eta} g^{(m)}\left(\xi+\left(a^{(m)}-a^{(m-1)}\right) \eta+C\right) d \eta+g^{(m-1)}(\xi) .
$$

By integration by parts, we represent the integral on the right-hand side in relation (3.15) in the form

$$
\begin{aligned}
& \int e^{\left(b^{(m-1)}-b^{(m)}\right) \eta} g^{(m)}\left(\xi+\left(a^{(m)}-a^{(m-1)}\right) \eta+C\right) d \eta \\
& =e^{\left(b^{(m-1)}-b^{(m)}\right) \eta} \tilde{g}^{(m)}\left(\xi+\left(a^{(m)}-a^{(m-1)}\right) \eta+C\right) \\
& \quad-\left(b^{(m-1)}-b^{(m)}\right) \int e^{\left(b^{(m-1)}-b^{(m)}\right) \eta} \tilde{g}^{(m)}\left(\xi+\left(a^{(m)}-a^{(m-1)}\right) \eta+C\right) d \eta,
\end{aligned}
$$

where $\tilde{g}^{(m)}$ is an arbitrary differentiable function defined via $g^{(m)}$. By returning to the representation (3.15) and then to the functions $v(t, x)$ and $w(t, x)$, we obtain the general solution of Eq. (3.11) as the sum of two functions

$$
w(t, x)=e^{-b^{(m)} t} f^{(m)}\left(x+a^{(m)} t+C^{(m)}\right)+e^{-b^{(m-1)} t} g^{(m-1)}\left(x+a^{(m-1)} t+C^{(m-1)}\right)
$$

provided that either $a^{(m)} \neq a^{(m-1)}$ or $b^{(m)} \neq b^{(m-1)}$, where $f^{(m)}$ and $g^{(m-1)}$ are arbitrary differentiable functions and $C^{(m-1)}$ and $C^{(m)}$ are arbitrary constants.

If $a^{(m)}=a^{(m-1)}$ and $b^{(m)}=b^{(m-1)}$, then from Eq. (3.14), we find the solution of Eq. (3.11) in the form

$$
w(t, x)=e^{-b^{(m)} t} f^{(m)}\left(x+a^{(m)} t+C^{(m)}\right)+t e^{-b^{(m)}} t g^{(m-1)}\left(x+a^{(m)} t+C^{(m-1)}\right) .
$$

By proceeding this way, we find the general solution $u$ of Eq. (3.1). Just as in the cases (3.17) and (3.18), its analytic form depends on the presence of equal coefficients $a^{(k)}$ and $b^{(k)}$, i.e., on the 
multiplicity of equal families of characteristics. In this connection, we refine the operator $\mathfrak{L}^{(m)}$ and rewrite it in a different form.

By $\mathfrak{K}$ we denote the set of integers from 1 to $m$; i.e., $\mathfrak{K}=\{1,2, \ldots, m\}$. We rewrite the operator $\mathfrak{L}^{(m)}$ in the form

$$
\tilde{\mathfrak{L}}^{(m)}=\prod_{k=1}^{p}\left(\partial_{t}-a^{(k)} \partial_{x}+b^{(k)}\right)^{r(k)},
$$

where $p$ and $r(k)$ are integer numbers in the set $\mathfrak{K}$ such that $\sum_{k=1}^{p} r(k)=m$. It follows from the above-performed considerations that the general solution of Eq. (3.19) has the form

$$
u(t, x)=\sum_{k=1}^{p} \sum_{s=1}^{r(k)} t^{s-1} e^{-b^{(k)} t} f^{(k s)}\left(x+a^{(k)} t+C^{(k s)}\right),
$$

where the $f^{(k s)}$ are arbitrary differentiable functions and the $C^{(k s)}$ are arbitrary real constants.

We have thereby proved the following assertion.

Theorem 3.1. The general solution of an arbitrary mth-order differential equation (3.1) in the case of two independent variables $t$ and $x$ with constant real coefficients $a^{(k)}$ and $b^{(k)}$ and with an operator $\mathfrak{L}^{(m)}$ of the form (3.19) can be represented by the sum (3.20) of $m$ terms, where $f^{(k s)}: \mathbb{R} \ni x+a^{(k)} t+C^{(k s)} \rightarrow f^{(k s)}\left(x+a^{(k)} t+C^{(k s)}\right) \in \mathbb{R}$ are arbitrary real functions and $(t, x) \in Q$.

Remark 3.1. Here the domain $Q$ can be a half-plane or the entire plane $\mathbb{R}^{2}$, depending on the definition of the functions $f^{(k s)}$.

\section{SOLUTION OF THE CAUCHY PROBLEM (3.1), (2.2)}

Consider Eq. (3.1) that has no multiple characteristics, or $b^{(k)} \neq b^{(j)}$ if $a^{(k)}=a^{(j)}, k \neq j$, $k, j \in \mathfrak{K}$. In this case, the general solution (3.20) has the form

$$
u(t, x)=\sum_{k=1}^{m} e^{-b^{(k)} t} f^{(k)}\left(x+a^{(k)} t+C^{(k)}\right) .
$$

We construct a function of the form (4.1) satisfying also condition (2.2). Let us compute the derivatives with respect to $t$ :

$$
\partial_{t}^{j} u(t, x)=\sum_{k=1}^{m} e^{-b^{(k)}} \sum_{s=0}^{j}(-1)^{j-s} A_{j}^{s}\left(b^{(k)}\right)^{j-s}\left(a^{(k)}\right)^{s} \partial_{t}^{s} f^{(k)}\left(x+a^{(k)} t+C^{(k)}\right),
$$

$j=0, \ldots, m-1, A_{j}^{s}=\frac{j !}{s !(j-s) !}, 0 !=1$.

By substituting the values of the derivatives (4.2) into condition (2.2), we obtain a system of equations for the functions $f^{(k)}\left(x+C^{(k)}\right), k=1, \ldots, m$,

$$
\sum_{k=1}^{m} \sum_{s=0}^{j}(-1)^{j-s} A_{j}^{s}\left(b^{(k)}\right)^{j-s}\left(a^{(k)}\right)^{s} d^{s} f^{(k)}\left(x+C^{(k)}\right)=d^{j} \varphi^{(j)}(x),
$$

$j=0, \ldots, m-1,\left(b^{(k)}\right)^{0}=1$. One should find the functions $f^{(k)}, k=1, \ldots, m$, from system $(4.3)$ in terms of the functions $\varphi^{(j)}, j=0, \ldots, m-1$.

4.1. First, consider the system in which all $b^{(k)}=0$ and $a^{(k)} \neq a^{(j)}$ for all $k \neq j$, where $j, k=1, \ldots, m$. In this case, system (4.3) acquires the form

$$
\sum_{k=1}^{m}\left(a^{(k)}\right)^{j} d^{j} f^{(k)}\left(x+C^{(k)}\right)=d^{j} \varphi^{(j)}(x), \quad j=0, \ldots, m-1 .
$$


By integrating equations in (4.4) for $j=1, \ldots, m-1$, we obtain the algebraic system of equations

$$
\sum_{k=1}^{m}\left(a^{(k)}\right)^{j} f^{(k)}\left(x+C^{(k)}\right)=\Phi^{(j)}(x, C), \quad j=0, \ldots, m-1,
$$

where

$$
\begin{aligned}
& \Phi^{(0)}(x, C)=\varphi^{(0)}(x), \\
& \Phi^{(j)}(x, C)=\varphi^{(j)}(x)+\sum_{s=0}^{j} \frac{x^{s}}{s !} C^{(j, s)}=\varphi^{(j)}(x)+P^{(j)}\left(x, C^{(j, s)}\right), \quad j=1, \ldots, m-1,
\end{aligned}
$$

and the $C^{(j, s)}$ are arbitrary constants that appear after the integration of the equations in system (4.4).

The determinant of the left-hand side of system (4.5) is the Vandermonde determinant (see $[17$, p. 50]); i.e.,

$$
\|A\|=\left|\begin{array}{ccc}
1 & \ldots & 1 \\
a^{(1)} & \ldots & a^{(m)} \\
\ldots & \ldots & \ldots \\
\left(a^{(1)}\right)^{m-1} & \ldots & \left(a^{(m)}\right)^{m-1}
\end{array}\right|=\prod_{1 \leq j<i \leq m}\left(a^{(i)}-a^{(j)}\right) .
$$

The matrix of the determinant (4.6) corresponds to the solution (4.1), i.e., to the case in which in Eq. (3.1) $a^{(k)} \neq a^{(j)}$ for $k \neq j, k, j \in\{1, \ldots, m\}$. Consequently, $\|A\| \neq 0$. Therefore, system (4.5) is solvable, and the functions $f^{(k)}$ are uniquely determined by the relations

$$
\begin{aligned}
& f^{(k)}\left(x+C^{(k)}\right)=\frac{1}{\|A\|}\left\|A^{(k)}(\varphi(x)+C(x))\right\|, \\
& \left\|A^{(k)}(\boldsymbol{\varphi}(x)+\boldsymbol{C}(x))\right\| \\
& =\left|\begin{array}{ccccccc}
1 & \ldots & 1 & \Phi^{(0)}(x, C) & 1 & \ldots & 1 \\
a^{(1)} & \ldots & a^{(k-1)} & \Phi^{(1)}(x, C) & a^{(k+1)} & \ldots & a^{(m)} \\
\ldots & \ldots & \ldots & \ldots & \ldots & \ldots & \ldots \\
\left(a^{(1)}\right)^{m-1} & \ldots & \left(a^{(k-1)}\right)^{m-1} & \Phi^{(m-1)}(x, C) & \left(a^{(k+1)}\right)^{m-1} & \ldots & \left(a^{(m)}\right)^{m-1}
\end{array}\right| \equiv \Delta(x, C) .
\end{aligned}
$$

Hence it follows that

$$
f^{(k)}\left(x+a^{(k)} t+C^{(k)}\right)=\frac{1}{\|A\|} \Delta\left(x+a^{(k)} t, C\right) .
$$

In formula (4.7), the $k$ th column

$$
\boldsymbol{\varphi}(x)+\boldsymbol{C}(x)=\left(\varphi^{(0)}(x), \varphi^{(1)}(x)+C^{(0,0)}, \ldots, \varphi^{(m-1)}(x)+P^{(m-1)}\left(x, C^{(m-1, s)}\right)\right)
$$

of the determinant $\Delta(x, C)$ is treated as the sum of two columns $\varphi(x)=\left(\varphi^{(0)}(x), \ldots, \varphi^{(m-1)}(x)\right)$ and $\boldsymbol{C}(x)=\left(0, C^{(0,0)}, C^{(1,0)}+x C^{(1,1)}, \ldots, P^{(m-1)}\left(x, C^{(m-1, s)}\right)\right)$. Then

$$
\left\|A^{(k)}(\boldsymbol{\varphi}(x)+\boldsymbol{C}(x))\right\|=\left\|A^{(k)}(\boldsymbol{\varphi}(x))\right\|+\left\|A^{(k)}(\boldsymbol{C}(x))\right\| .
$$

By taking into account relation (4.9) and the representation (4.1), for the special case in which Eq. (3.1) has no multiple characteristics and $b^{(k)}=0, k=1, \ldots, m$, we write out the solution of problem (3.1), (2.2) via the Vandermonde determinant and a determinant of the Vandermonde type as the sum

$$
u(t, x)=\frac{1}{\|A\|} \sum_{k=1}^{m}\left\|A^{(k)} \boldsymbol{\varphi}\left(x+a^{(k)} t\right)\right\|+\frac{1}{\|A\|} \sum_{k=1}^{m}\left\|A^{(k)} \boldsymbol{C}\left(x+a^{(k)} t\right)\right\| .
$$


Consider the sum of determinants, which are modified Vandermonde determinants $\|A\|$. That determinant is obtained from the Vandermonde determinant by the replacement of the $k$ th column by the column consisting of zeros except for the entry $a^{(k) s}$ in the intersection of the $k$ th column and the $(j+1)$ st row. Thus, consider the sum of such determinants $B^{(j+1, m)}\left(a^{(k) s}\right)$ of order $m$, i.e.,

$$
\begin{aligned}
& \sum_{k=1}^{m} B^{(j+1 ; m)}\left(a^{(k) s}\right)=\left|\begin{array}{cccc}
0 & 1 & \ldots & 1 \\
\ldots & \ldots & \ldots & \ldots \\
a^{(1) s} & a^{(2) j} & \ldots & a^{(m) j} \\
\ldots & \ldots & \ldots & \ldots \\
0 & a^{(2) m-1} & \ldots & a^{(m) m-1}
\end{array}\right|+\left|\begin{array}{cccc}
1 & 0 & \ldots & 1 \\
\ldots & \ldots & \ldots & \ldots \\
a^{(1) j} & a^{(2) s} & \ldots & a^{(m) j} \\
\ldots & \ldots & \ldots & \ldots \\
a^{(1) m-1} & 0 & \ldots & a^{(m) m-1}
\end{array}\right|+\ldots \\
& +\left|\begin{array}{ccccc}
1 & \ldots & 0 & \ldots & 1 \\
\ldots & \ldots & \ldots & \ldots & \ldots \\
a^{(1) j} & \ldots & a^{(k) s} & \ldots & a^{(k) j} \\
\ldots & \ldots & \ldots & \ldots & \ldots \\
a^{(1) m-1} & \ldots & 0 & \ldots & a^{(m) m-1}
\end{array}\right|+\cdots+\left|\begin{array}{cccc}
1 & \ldots & 1 & 0 \\
\ldots & \ldots & \ldots & \ldots \\
a^{(1) j} & \ldots & a^{(m-1) j} & a^{(m) s} \\
\ldots & \ldots & \ldots & \ldots \\
a^{(1) m-1} & \ldots & a^{(m-1) m-1} & 0
\end{array}\right| .
\end{aligned}
$$

Lemma 4.1. The relation

$$
\sum_{k=1}^{m} B^{(j+1 ; m)}\left(a^{(k) s}\right)=0, \quad s=0, \ldots, j-1,
$$

holds for arbitrary distinct real values $a^{(i)}, i=1, \ldots, m\left(a^{(r)} \neq a^{(p)}, r \neq p, r, p=1, \ldots, m\right)$.

Proof. By expanding each determinant $B^{(j+1 ; m)}\left(a^{(k) s}\right)$ in entries of the $k$ th column, we obtain the relation

$$
\sum_{i=1}^{m} B^{(j+1 ; m)}\left(a^{(k) s}\right)=\sum_{i=1}^{m}(-1)^{k+j+1} a^{(k) s} M^{(j+1, k ; m-1)},
$$

where the $M^{(j+1, k ; m-1)}$ are complementary minors of order $m-1$ for the entries $a^{(k) s}$ on the intersection of the $(j+1)$ st row and the $k$ th column in the determinants $B^{(j+1 ; m)}\left(a^{(k) s}\right)$. By the assumption of the lemma $(s \in\{0, \ldots, j-1\})$, the $(s+1)$ st row in the minor $M^{(j+1, k ; m-1)}$ consists of the entries $a^{(r) s}, r \in\{1, \ldots, m\}, r \neq k$. We multiply that row by the entry $a^{(k) s}$. As a result, we obtain the minor $\tilde{M}^{(j+1, k ; m-1)}$, which differs from $M^{(j+1, k ; m-1)}$ by the $(s+1)$ st row, where the entries $a^{(r) s}$ are replaced by the entries $a^{(k) s} a^{(r) s}, r \in\{1, \ldots, m\}, r \neq k$. We expand each of the minors $\tilde{M}^{(j+1, k ; m-1)}$ in a sum in the entries $a^{(k) s} a^{(r) s}$ of the $(s+1)$ st row. As a result, we obtain the expansion

$$
\tilde{M}^{(j+1, k ; m-1)}=\sum_{\substack{r \in\{1, \ldots, m\} \\ r \neq k}}(-1)^{s+p+1} a^{(k) s} a^{(r) s} M^{(s+1, p ; m-2)}\left(a^{(k) s} a^{(r) s}\right),
$$

where $M^{(s+1, p ; m-2)}\left(a^{(k) s} a^{(r) s}\right)$ are the complementary minors in $\tilde{M}^{(j+1, k ; m-1)}$ with respect to the entries $a^{(k) s} a^{(r) s}$ in the intersection of the $(s+1)$ st row and the $p$ th column; here $p=r-1$ if $r>k$ and $p=r$ if $r<k$. Next, we have $a^{(k) s} M^{(j+1, k ; m-1)}=\tilde{M}^{(j+1, k ; m-1)}$. By substituting the minors (4.14) into relation (4.12), we obtain

$$
\sum_{i=1}^{m} B^{(j+1 ; m)}\left(a^{(k) s}\right)=\sum_{k=1}^{m} \sum_{\substack{r \in\{1, \ldots, m\} \\ r \neq k}}(-1)^{k+j+s+p} a^{(k) s} a^{(r) s} M^{(s+1, p ; m-2)}\left(a^{(k) s} a^{(r) s}\right) .
$$

By virtue of the definition of $p$, we have

$$
(-1)^{k+j+s+p} a^{(k) s} a^{(r) s}=-(-1)^{k+j+s+p} a^{(r) s} a^{(k) s}
$$


and

$$
M^{(s+1, p ; m-2)}\left(a^{(k) s} a^{(r) s}\right)=M^{(s+1, p ; m-2)}\left(a^{(r) s} a^{(k) s}\right) .
$$

This, together with relation (4.15), implies the assertion of Lemma 4.1.

By $C^{m}(\mathbb{R})$ we denote the set of functions that are continuous, continuously differentiable up to order $m$, and defined on the entire set $\mathbb{R}$ of real numbers.

Theorem 4.1. If the function $\varphi^{(k)}$ belongs to $C^{m}(\mathbb{R}), k=0, \ldots, m-1$, and Eq. (3.1) is strictly hyperbolic and does not contain terms with lower derivatives $\left(a^{(k)} \neq a^{(j)}, k \neq j, b^{(k)}=0, k, j \in\right.$ $\{1, \ldots, m\})$, then there exists a unique solution $u$ of problem $(3.1),(2.2)$ in the class $C^{m}(\bar{Q})$, and this solution is defined by the formula

$$
u(t, x)=\frac{1}{\|A\|} \sum_{k=1}^{m}\left\|A^{(k)} \boldsymbol{\varphi}\left(x+a^{(k)} t\right)\right\|,
$$

where $C^{m}(\bar{Q})$ is the set of functions continuous and continuously differentiable with respect to $t$ and $x$ up to the order $m$ in the closure $\bar{Q}$ of the domain $Q \subset \mathbb{R}^{2}$.

Proof. Under assumptions of the theorem, the solution of problem (3.1), (2.2) has the form (4.10). The second term in relation (4.10) can be represented in the form of the expansion in $C^{(j-1, s)}$, $j=1, \ldots, m-1, s=0, \ldots, j-1$, and in powers of $x$ and $t$. As a result, we obtain the expansion

$$
\sum_{k=1}^{m}\left\|A^{(k)} \boldsymbol{C}\left(x+a^{(k)} t\right)\right\|=\sum_{j, s, r, p} \mathcal{K}\left(x^{r}, t^{p}, C^{(j-1, s)}\right) B\left(x^{r}, t^{p}, C^{(j-1, s)}\right) x^{r} t^{p} C^{(j-1, s)},
$$

where the sums of the coefficients $B\left(x^{r}, t^{p}, C^{(j-1, s)}\right)$ are sums of minors of the form (4.11). By Lemma 4.1, all sums $B\left(x^{r}, t^{p}, C^{(j-1, s)}\right)$ are zero. Consequently, the solution $u$ of problem (3.1), (2.2) contains no term depending on arbitrary constants $C^{(j-1, s)}$ and is uniquely determined by formula (4.16). The proof of Theorem 4.1 is complete.

4.2. Now consider a strictly hyperbolic equation (3.1) with lower derivatives, i.e., the case in which all or some of $b^{(k)}$ are nonzero, $k \in\{1, \ldots, m\}$.

In this case, the general solution of Eq. (3.1) is given by formula (4.1), and the derivatives with respect to $t$ are defined by relation (4.2). To determine the solution of problem (3.1), (2.2), we substitute the solution (4.1) into condition (2.2) or into system (4.3). From (4.3), for the unknown functions $f^{(k)}\left(x+C^{(k)}\right)$, we obtain the system of equations

$$
\begin{aligned}
& \sum_{k=1}^{m} f^{(k)}\left(x+C^{(k)}\right)=\varphi^{(0)}(x), \\
& \sum_{k=1}^{m}\left(a^{(k)}\right)^{j} d^{j} f^{(k)}\left(x+C^{(k)}\right) \\
& \quad+\sum_{k=1}^{m} \sum_{s=0}^{j-1}(-1)^{j-s} A_{j}^{s} \sum_{k=1}^{m}\left(b^{(k)}\right)^{j-s}\left(a^{(k)}\right)^{s} d^{s} f^{(k)}\left(x+C^{(k)}\right)=d^{j} \varphi^{(j)}(x),
\end{aligned}
$$

$j=1, \ldots, m-1$. We integrate each equation in (4.18) except for the first one $j$ times. Then we obtain the system

$$
A \boldsymbol{f}(x+C)+K \boldsymbol{f}(x+C)=\boldsymbol{\varphi}(x)+\boldsymbol{C}(x),
$$

where

$$
\begin{aligned}
\boldsymbol{f}(x+C) & =\left(f^{(1)}\left(x+C^{(1)}\right), \ldots, f^{(m)}\left(x+C^{(m)}\right)\right), \quad \boldsymbol{\varphi}(x)=\left(\varphi^{(0)}(x), \varphi^{(1)}(x), \ldots, \varphi^{(m-1)}(x)\right), \\
\boldsymbol{C}(x) & =\left(0, C^{(0,0)}, C^{(1,0)}+x C^{(1,1)}, \ldots, C^{(m-2,0)}+\cdots+\frac{x^{(m-2)}}{(m-2) !} C^{(m-2, m-2)}\right),
\end{aligned}
$$

DIFFERENTIAL EQUATIONS $\quad$ Vol. 48 No. $5 \quad 2012$ 
$A$ is the matrix of the Vandermonde determinant of the form (4.6), and $K=\left(K^{(i, j+1)}\right)$ is a matrix of integral operators with Volterra integration operators. The entries of the matrix $K=\left(K^{(i, j+1)}\right)$, where $i=1, \ldots, m$ is the index of the column and $j=0, \ldots, m-1$ is the number of the row, have the form

$$
\begin{gathered}
K^{(i, 1)} \equiv 0, \quad i=1, \ldots, m, \quad K^{(i, 2)}=-b^{(1)} \int_{0}^{x} d z, \\
K^{(i, j+1)}=\sum_{s=0}^{j-1}(-1)^{j-s} A_{j}^{s}\left(b^{(i)}\right)^{j-s}\left(a^{(i)}\right)^{s} \frac{1}{(j-1-s) !} \int_{0}^{x}(x-z)^{j-1-s} d z, \\
i=1, \ldots, m, \quad j=2, \ldots, m-1 .
\end{gathered}
$$

Consider system (4.19). Since the operator $K$ is a set of integral operators, it follows that the successive approximation method can be applied to Eqs. (4.19). For the zero approximation, we take

$$
\boldsymbol{f}_{0}(x+C)=\left(f_{0}^{(1)}\left(x+C^{(1)}\right), \ldots, f_{0}^{(m)}\left(x+C^{(m)}\right)\right),
$$

where the functions $f_{0}^{(k)}\left(x+C^{(k)}\right)$ satisfy the equation

$$
A f_{0}(x+C)=\varphi(x)+C(x) .
$$

Since $\|A\|=\prod_{1 \leq j<i<m}\left(a^{(i)}-a^{(j)}\right) \neq 0$, it follows from Theorem 4.1 that system (4.20) has a unique solution $\boldsymbol{f}_{0}(x+\bar{C})$. By substituting this solution as the zero approximation into Eq. (4.19), we obtain the new equation

$$
A \boldsymbol{f}_{1}(x+C)=\boldsymbol{\varphi}(x)-K \boldsymbol{f}_{0}(x+C)+C(x)=\boldsymbol{\varphi}_{1}(x)+C(x) .
$$

By solving Eq. (4.21), we obtain the next approximation $\boldsymbol{f}_{1}(x+C)$, which is also uniquely determined. The approximation $\boldsymbol{f}_{2}(x+C)$ is found from the equation

$$
A \boldsymbol{f}_{2}(x+C)=\varphi_{1}(x)-K \boldsymbol{f}_{1}(x+C)+C(x)=\varphi_{2}(x)+C(x) .
$$

By proceeding this way, we obtain a sequence $\left\{\boldsymbol{f}_{n}(x+C)\right\}_{n=1}^{\infty}$ of approximations $\boldsymbol{f}_{n}(x+C)$ satisfying the system of equations

$$
A \boldsymbol{f}_{n}(x+C)=\boldsymbol{\varphi}_{n-1}(x)-K \boldsymbol{f}_{n-1}(x-C)+C(x)=\boldsymbol{\varphi}_{n}(x)+C(x) .
$$

The subsequent approximations in system (4.22) are defined via the previous ones in accordance with formulas (4.7) and (4.8). Hence one can find that the components of the vector $\boldsymbol{f}_{n}(x+C)$ differ from $\boldsymbol{f}_{n-1}(x+C)$ by terms of values of operators of the multiple applications of integrals of Volterra operators to elements of the vector $\varphi$. It is known that the value of the operator representing the $n$th power of the Volterra operator applied to components of the vector $\varphi(x)$ in an arbitrary bounded domain tends to zero as $n \rightarrow \infty$, and the series representing the approximations $f_{n}^{(k)}(x+C)$ or $f_{n}^{(k)}\left(x+a^{(k)} t+C\right)$ of the vectors $\boldsymbol{f}_{n}(x+C)$ in these domains are convergent uniformly due to factorials and are continuous functions.

Since the $\varphi^{(j)}(j=0, \ldots, m-1)$ are continuous functions, it follows that the functions $f_{n}^{(k)}(k=$ $1, \ldots, m)$ converge uniformly to a continuous function $f^{(k)}, \boldsymbol{f}(x+C)=\left(f^{(1)}(x+C), \ldots, f^{(m)}(x+C)\right)$, in an arbitrary bounded domain as $n \rightarrow \infty$. Since each approximation $\boldsymbol{f}_{n}(x+C)$ is a solution of Eq. (4.22) and, by virtue of Lemma 4.1, is uniquely determined, it follows that the vector function $\boldsymbol{f}(x+C)$ is uniquely determined as well.

By passing in Eq. (4.22) to the limit as $n \rightarrow \infty$, we find that the constructed vector function $\boldsymbol{f}(x+C)$ satisfies Eq. (4.19).

Consider the resultant functions $f^{(k)}$ as functions of the arguments $x+a^{(k)} t+C$ and substitute them into (4.1). The function $u$ defined in this way by relation (4.1) via the above-mentioned functions $f^{(k)}$ treated as solutions of system (4.19) is a solution of problem (3.1), (2.2) for the strictly hyperbolic equation (3.1). We state the obtained result in the form of a theorem. 
Theorem 4.2. If the $\varphi^{(k)}, k=0, \ldots, m-1$, are functions of the class $C^{m}(\mathbb{R})$ and Eq. (3.1) is strictly hyperbolic, then there exists a unique solution of problem $(3.1),(2.2)$ in the class $C^{(m)}(Q)$, which is given by formula (4.1), where the $f^{(k)}, k=1, \ldots, m$, are solutions of system (4.19).

4.3. Now consider the Cauchy problem (2.1), (2.2) for the inhomogeneous strictly hyperbolic equation (2.1). Since the considered problem is linear, it follows that its solution $u$ can be represented by the sum of two functions

$$
u(t, x)=u^{(0)}(t, x)+u^{(1)}(t, x),
$$

where $u^{(0)}$ is the solution of problem (3.1), (2.2) given by formula (4.1). The function $u^{(1)}$ is a solution of the inhomogeneous equation (2.1) satisfying the homogeneous Cauchy conditions

$$
\left.\partial_{t}^{j} u^{(1)}\right|_{t=0}=0, \quad x \in \mathbb{R}, \quad j=0, \ldots, m-1 .
$$

To find the solution of problem (2.1), (4.24), we use the solution of problem (3.1), (2.2). Let $w(t, \tau, x)$ be the solution of the Cauchy problem

$$
\begin{aligned}
& \prod_{k=1}^{m}\left(\partial_{t}-a^{(k)} \partial_{x}+b^{(k)}\right) w(t, \tau, x)=0, \\
& \left.\partial_{t}^{j} w(t, \tau, x)\right|_{t=0}=0, \quad j=0, \ldots, m-2,\left.\quad \partial_{t}^{m-1} w(t, \tau, x)\right|_{t=0}=f(\tau, x) .
\end{aligned}
$$

Set

$$
\varphi^{(m)}(\tau, x)=\frac{1}{(m-2) !} \int_{0}^{x}(x-z)^{m-2} f(\tau, z) d z, \quad \varphi(\tau, x)=\left(0, \ldots, 0, \varphi^{(m)}(\tau, x)\right) .
$$

By using these functions and formula (4.1), we find the solution of problem (4.25), (4.26). In a special case, after performing the computations for $b^{(k)}=0, k=1, \ldots, m$, we obtain the function

$$
w(t, \tau, x)=\sum_{k=1}^{m} \varphi^{m}\left(\tau, x+a^{(k)} t\right)\left(\prod_{\substack{i=1 \\ i \neq k}}^{m}\left(a^{(k)}-a^{(i)}\right)\right)^{-1} .
$$

The function $u^{(1)}(t, x)$ represented by the relation

$$
u^{(1)}(t, x)=\int_{0}^{t} w(t-\tau, \tau, x) d \tau
$$

via $w(t, \tau, x)$ is a solution of problem $(2.1),(4.24)$. This can readily be verified by a straightforward substitution into Eq. (2.1) and conditions (4.24) with regard of the fact that, in this case, $w$ is a solution of problem (4.25), (4.26).

Likewise, if $b^{(k)}=0, k=1, \ldots, m$, then, by combining formulas (4.27) and (4.28), in the case of the homogeneous differential operator $\mathfrak{L}^{(m)}$, we obtain the representation of the function $u^{(1)}$ in the form

$$
u^{(1)}(t, x)=\frac{1}{(m-2) !} \int_{0}^{t} \sum_{k=1}^{m}\left(\prod_{\substack{i=1 \\ i \neq k}}^{m}\left(a^{(k)}-a^{(i)}\right)\right)^{-1} \int_{0}^{x+a^{(k)}(t-\tau)}\left(x+a^{(k)}(t-\tau)-z\right)^{m-2} f(\tau, z) d z d \tau .
$$

By $C^{1}(\bar{Q})$ we denote the set of continuous and continuously differentiable functions defined on $\bar{Q}$. We have thereby proved the following assertion. 
Theorem 4.3. If Eq. (2.1) is strictly hyperbolic, the functions $\varphi^{(k)}, k=0, \ldots, m-1$, belong to the class $C^{(k)}(\mathbb{R})$, and $f \in C^{1}(\bar{Q})$, then there exists a unique solution u of problem $(2.1),(2.2)$ in the class $C^{m}(\bar{Q})$, whose closed form is given by formulas (4.23), (4.1), (4.28), (4.7), and (4.8).

\section{REFERENCES}

1. Emmanuele Di Benedetto, Partial Differential Equations, Boston, 1995.

2. Tikhonov, A.N. and Samarskii, A.A., Uravneniya matematicheskoi fiziki (Equations of Mathematical Physics), Moscow, 2004.

3. Matveev, N.M., Metody integrirovaniya obyknovennykh differentsial'nykh uravnenii (Integration Methods for Ordinary Differential Equations), Moscow, 1967.

4. Erugin, N.P., Kniga dlya chteniya po obshchemu kursu differentsial'nykh uravnenii (A Reader for a General Course in Differential Equations), Minsk: Nauka i Tekhnika, 1972.

5. Tran Duc Van, Mikio Tsuji, and Nguen Duy Thai Son, The Characteristic Method and Its Generalizations for First-Order Nonlinear Partial Differential Equations, in Monogr. Surv. Pure Appl. Math., Boca Raton, 2000, no. 101.

6. Gårding, L., Cauchy's Problem for Hyperbolic Equations, Chicago, 1957. Translated under the title Zadacha Koshi dlya giperbolicheskikh uravnenii, Moscow: Inostrannaya literatura, 1961.

7. Petrovskii, I.G., Über das Cauchysche Problem für System von partiellen Differentialgleichungen, Mat. Sb., 1937, no. 2 (44), pp. 815-870.

8. Petrovskii, I.G., Sur l'analyticité des solutions des systèmes d'équations différentielles, Mat. Sb., 1939, no. 5 (47), pp. 3-70.

9. Roitberg, Ya.A., Cauchy Problem, Boundary and Mixed Problems for General Hyperbolic Systems in a Full Scale of Sobolev-Type Spaces, Nelin. Granichn. Zad., 1990, no. 2, pp. 93-98.

10. Roitberg, Ya.A., The Cauchy Problem for General Hyperbolic Systems in a Complete Scale of SobolevType Spaces, Dokl. Akad. Nauk SSSR, 1991, vol. 316, no. 2, pp. 300-304.

11. Roitberg, Ya.A., A Boundary and Mixed Problems for General Hyperbolic Systems in a Complete Scale of Spaces of Sobolev Type, Dokl. Akad. Nauk SSSR, 1991, vol. 318, no. 4, pp. 820-824.

12. Leray, J., Lectures on Hyperbolic Equations with Variable Coefficients, Princeton, 1952.

13. Leray, J., Hyperbolic Differential Equations, Princeton, 1955. Translated under the title Giperbolicheskie differentsial'nye uravneniya, Moscow: Nauka, 1984.

14. Torchinsky, A., The Fourier Transform and the Wave Equation, arXiv: 0904.3252v1 [math.AP] 21 Apr 2009.

15. Mamadaliev, N.K., On the Representation of the Solution of a Modified Cauchy Problem, Sibirsk. Mat. Zh., 2000, vol. 41, no. 5, pp. 1087-1097.

16. Kragler, R., The Method of Inverse Differential Operators Applied for the Solution of PDEs, in Computer Algebra Systems in Teaching and Research. Differential Equations, Dynamical Systems and Celestial Mechanics, Gadomski, L., Jakubiak, M., and Prokopenya, A.N., Eds., Siedlce, 2011, pp. 79-95.

17. Kurosh, A.G., Kurs vysshei algebry (The Course of Higher Algebra), Moscow, 1965. 